\title{
Advances in Video Coding for Broadcast Applications
}

\author{
Susanna Spinsante, ${ }^{1}$ Ennio Gambi, ${ }^{1}$ Lorenzo Ciccarelli, ${ }^{2}$ Andrea Lorenzo Vitali, ${ }^{3}$ \\ Jorge Sastre Martínez, ${ }^{4}$ and Paul Salama ${ }^{5}$ \\ ${ }^{1}$ Department of Biomedic Engineering, Electronics and Telecommunications, Università Politecnica delle Marche, \\ Via Brecce Bianche 12, 60131 Ancona, Italy \\ ${ }^{2}$ Video Broadcasting Coding, Basingstoke, UK \\ ${ }^{3}$ STMicroelectronics, Research \& Innovation, 20041 Milano, Italy \\ ${ }^{4}$ Image and Video Processing Group (GPIV), Multimedia Applications and Telecommunications Institute (iTEAM), \\ Technical University of Valencia, Camino de Vera, 46022 Valencia, Spain \\ ${ }^{5}$ Electrical and Computer Engineering, Indiana University-Purdue University, 723 West Michigan Street, SL160, \\ Indianapolis, IN 46202, USA
}

Correspondence should be addressed to Susanna Spinsante, s.spinsante@univpm.it

Received 31 March 2009; Accepted 31 March 2009

Copyright ( 2009 Susanna Spinsante et al. This is an open access article distributed under the Creative Commons Attribution License, which permits unrestricted use, distribution, and reproduction in any medium, provided the original work is properly cited.

The growing diffusion of new services, like mobile television and video communications, based on a variety of transmission platforms (3G, WiMax, DVB-S/T/H, DMB, DTMB, Internet, etc.), emphasizes the need of advanced video coding techniques able to meet the requirements of both the receiving devices and the transmission networks. In this context, scalable and layered coding techniques represent a promising solution when aimed at enlarging the set of potential devices capable of receiving video content. Video encoders' configuration must be tailored to the target devices and services, that range from high definition, for powerful high-performance home receivers, to video coding for mobile handheld devices. Encoder profiles and levels need to be tuned and properly configured to get the best tradeoff between resulting quality and data rate, in such a way as to address the specific requirements of the delivery infrastructure. As a consequence, it is possible to choose from the entire set of functionalities of the same video coding standard in order to provide the best performance for a specified service. Among the most recent video coding standards, the ITU-T H.264/AVC offers a wide set of configurations, that make it able to address several different services, ranging from video streaming, to DVB$\mathrm{T} / \mathrm{H}$ broadcasting, to videoconferencing over IP networks.

This special issue aims to present state-of-the-art research and developing activities contributing to all aspects of video coding solutions focused on broadcast applications. The Call-for-Papers for this special issue resulted in the excellent submissions from around the world in both quality and quantity. After two rounds of careful reviews from about fifty experts in related fields, five papers were selected to be included in this special issue covering major topics, from coding related issues to error resilience and protection and to statistical multiplexing, thus providing a comprehensive overview on the area.

The first paper entitled "New adaptive algorithms for GOP size control with return channel suppression in WynerZiv video coding" by C. Yaacoub et al. presents a novel algorithm for adaptive GOP size control in distributed Wyner-Ziv video coding, where key frames are intra coded according to the H.264/AVC standard. In order to avoid the use of a feedback channel, which is generally missing in broadcasting applications, theoretical calculations are used to estimate the bit rate necessary for successful decoding of Wyner-Ziv frames. The proposed system is also able to automatically switch to H.264 intra coding mode in image regions where it outperforms Wyner-Ziv encoding, to improve the overall PSNR with respect to pure H.264 intra coding, and fixed-GOP Wyner-Ziv coding.

The second paper, entitled "Intra skip in inter frame coding of H.264/AVC" by H. Su, deals with a novel mathematical model to skip intra mode predictions in inter frames coding according to H.264/AVC. The model is basically built around a weighted-coefficient function used to set a proper threshold, which, in its turn, affects the skipping of intra partitions evaluation. The aim of the proposed algorithm 
is to minimize the complexity of inter coding, by cutting down encoding time, while facing a very minor increase of the resulting bit rate, thus making H.264/AVC codecs more suitable to real time applications. Moreover, the proposed solution can be applied in conjunction with any proposed "fast" inter and intra methods relying on inter partition mode decision, motion search algorithms, and fast intra algorithms.

Moving from specific H.264/AVC coding issues to the problems related to broadcast video transmission, the third paper entitled "An adaptive systematic lossy error protection scheme for broadcast applications based on frequency filtering and unequal picture protection", by M. Ramon et al., discusses an adaptive systematic lossy error protection scheme in which the Wyner-Ziv stream is obtained by means of frequency filtering in the transform domain. Besides that, error resilience may vary adaptively, according to the characteristics of the compressed video. By applying the proposed solution, a graceful degradation of the reconstructed video quality may be obtained even in presence of increasing transmission errors, and the proposed scheme is shown to provide better performance than other solutions, such those based on coarser quantization.

Error resilience in the context of video streaming is the topic discussed by the fourth paper entitled "Adaptive error resilience for video streaming", by L. R. Siruvuri et al. It is well known that compressed video sequences may be strongly affected by channel errors, and suitable channel coding schemes may reduce the impact of errors on the quality of the decoded video. The paper suggests a solution to adapt the number of Reed-Solomon parity symbols used to protect a compressed video sequence against channel errors, in order to minimize the impact of the redundant bits on the available bandwidth. This paper spans the reference broadcasting scenario, by assuming the availability of a return channel through which feedbacks from the client are used to properly tune the amount of parity bits. As a matter of fact, the possibility of a return channel is already foreseen in some broadcasting systems, such as the Digital Video Broadcasting Return Channel Satellite, a possible scenario in which the proposed solution could find application.

The last paper included in this special issue, "Statistical time-frequency multiplexing of HD video traffic in DVBT2" by M. Rezaei et al. presents a model for describing High Definition video traffic, which is used to evaluate the performance of statistical multiplexing of HD broadcast services over DVB-T2. New features introduced by DVBT2 with respect to DVB-T, such as a two-dimensional multiplexing of broadcast services in time and frequency domains, and a time-frequency slicing transmission scheme, allow to increase the flexibility of service multiplexing, which may be of vital importance when dealing with $\mathrm{HD}$ video broadcasting. The model presented in the paper is able to simulate a wide range of synthetic video bit streams with practical and statistical metrics of interest; performance evaluation of DVB-T2 shows the possibility of getting improved performance in terms of bandwidth efficiency, end-to-end delay, and video quality for the broadcast system.
As we conclude this overview, we would like to express our sincere gratitude to all the reviewers for their timely and insightful comments on the submitted manuscripts, which made this special issue possible.

Susanna Spinsante Ennio Gambi

Lorenzo Ciccarelli

Andrea Lorenzo Vitali

Jorge Sastre Martínez

Paul Salama 

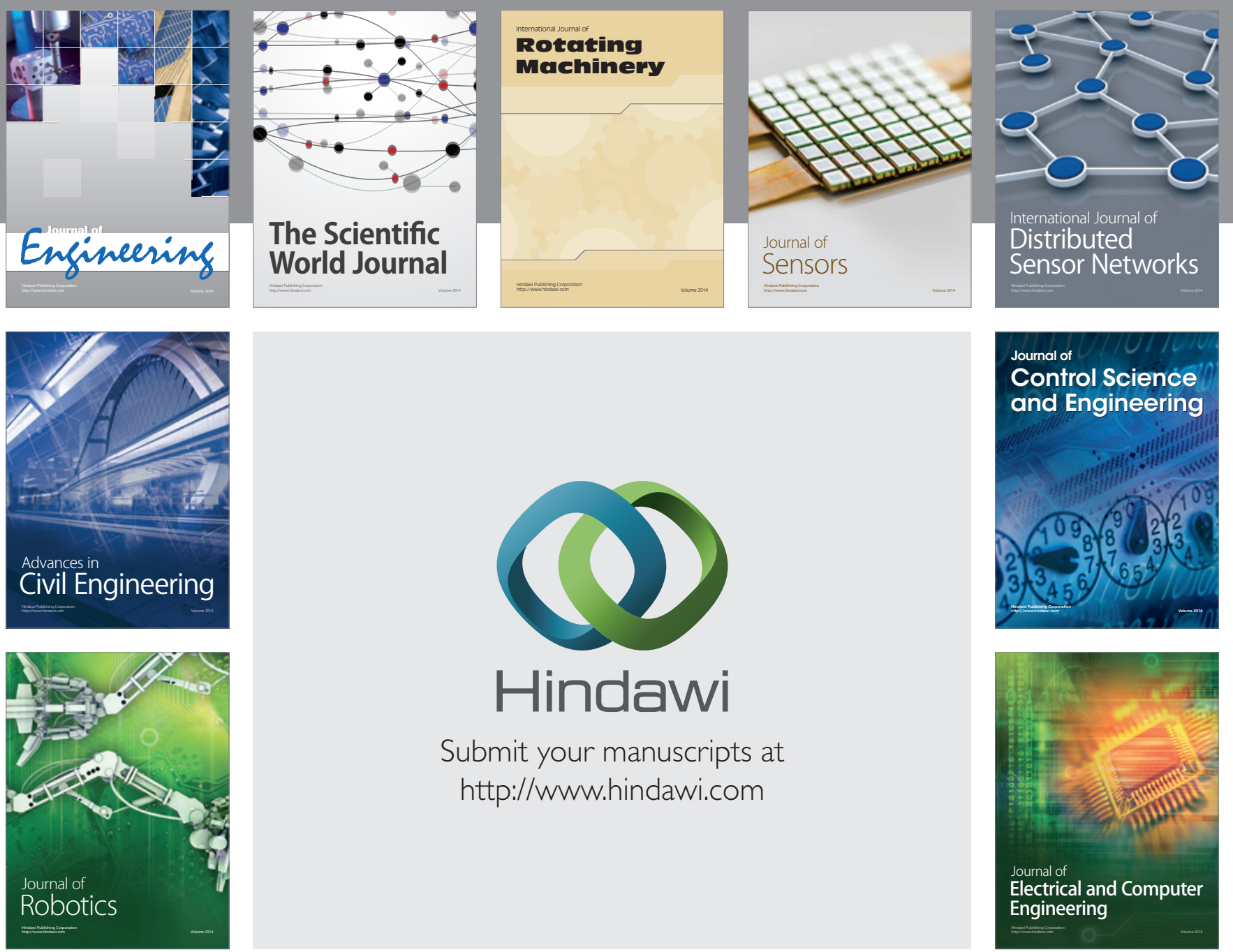

Submit your manuscripts at

http://www.hindawi.com
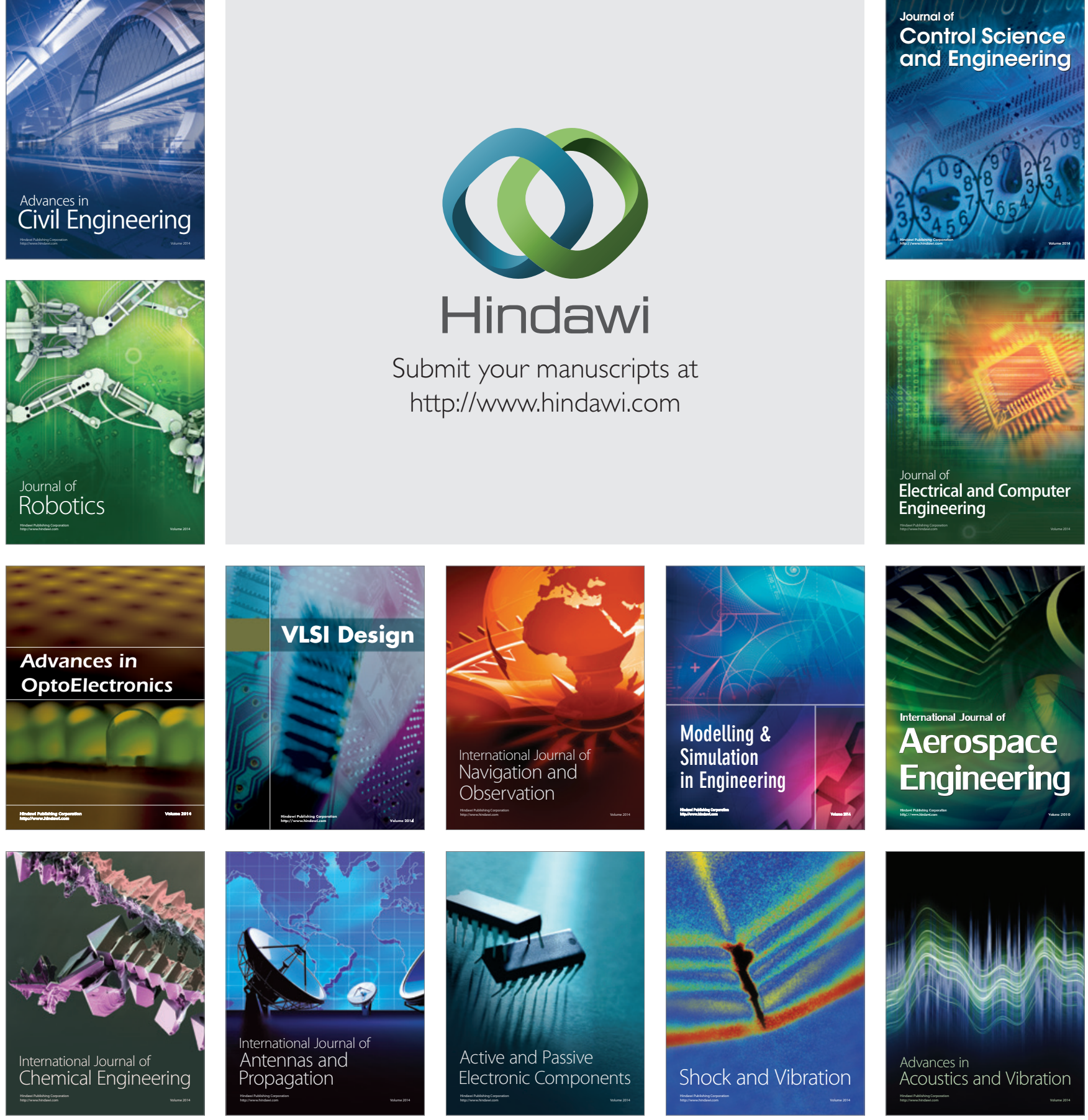